\title{
Reed Solomon Codes for Molecular Communication with Full Absorption Receiver
}

\author{
Maheshi B. Dissanayake, Yansha Deng, Arumugam Nallanathan, E. M. N. Ekanayake, and Maged Elkashlan
}

\begin{abstract}
Molecular communication (MC) has recently emerged as a novel paradigm for nano-scale communication utilizing molecules as information carriers. In diffusion-based molecular communication, the system performance is constrained by the inter-symbol-interference (ISI) caused by crossover of information carrying molecules in consecutive bits. To cope with this, we propose the Reed-Solomon (RS) codes as an error recovery tool, to improve the transmission reliability in diffusionbased MC systems. To quantify the performance improvement due to RS codes, we derive the analytical expression for the approximate bit error probability (BEP) of the diffusion-based MC system with the full absorption receiver. We further develop the particle-based simulation framework to simulate the proposed system with RS code to verify the accuracy of our derived analytical results. Our results show that, as the number of molecules per bit increases, the BEP of the system with RS codes exhibits a substantial improvement than that of non-coded systems. Furthermore, the BEP of the proposed system with RS codes can be greatly improved by increasing the minimum distance of the codeword.
\end{abstract}

Index Terms-Molecular communication, error correction codes, Reed Solomon codes, particle-based simulation,

\section{INTRODUCTION}

W ITH the advancements in nano-technology, new multidisciplinary research on communication between bionanomachines via chemical signals is emerging [1]. This new paradigm, namely Molecular Communication (MC), adopts biologically-inspired techniques for information transmission among nano-machines. The nature inspired molecular communication can be observed in calcium signalling among cells, and Deoxyribonucleic Acid (DNA) signalling among DNA segments [2] In this paper, we concentrate on the molecular communication via diffusion (MCvD) [3] to provide the baseline for the communication design, which will serve as an important foundation for designing more complicated $\mathrm{MC}$ systems with drift. In MCvD, the transmitter modulates the information onto the physical properties of the information carrying molecules. Once emitted by the transmitter, the molecules diffuse in the medium and certain portion of the

Manuscript received Sep. 6, 2016; revised Jan. 9, 2017 and Feb. 9, 2017; accepted Feb. 16, 2017. The editor coordinating the review of this manuscript and approving it for publication was Prof. Nghi Tran.

Maheshi B. Dissanayake is with the Department of Electrical and Electronic Engineering, Faculty of Engineering, University of Peradeniya, Sri Lanka and part of this work was carried out while at King's College London, London, WC2R 2LS, UK. (e-mail: maheshid@ee.pdn.ac.lk).

E.M.N. Ekanayake is with the Faculty of Engineering, University of Peradeniya, Sri Lanka. (e-mail: neka@ee.pdn.ac.lk).

Y. Deng and A. Nallanathan are with King's College London, London, WC2R 2LS, UK (email:yansha.deng,arumugam.nallanathan@kcl.ac.uk).

M. Elkashlan is with Queen Mary University of London, London E1 4NS, UK (email: maged.elkashlan@qmul.ac.uk). transmitted molecules arrive at the receiver during the current bit interval. The receiver then demodulates the transmitted information using the number of molecules captured during that bit interval. Due to the random walk of each molecule in the fluid medium, there exists high probability of crossover between the molecules emitted in neighboring bits. These molecules which arrive during different bit intervals, result in the so-called inter-symbol-interference (ISI) [4]. Note that ISI is regarded as one of the fundamental bottlenecks of MC systems that degrades the system reliability.

In the literature, there exist many different approaches for ISI mitigation in MC. As summarized in [1], borrowing from traditional communication system designs, equalization techniques like decision feedback filter technique, transmitterbased novel modulation techniques such as molecular transition shift keying and Molecular ARray-based COmmunication (MARCO), and energy efficient transmitter and receiver-side signal power adjustment methods have been proposed as ISI mitigation techniques for MC environment. Although, some of these techniques increase the complexity of the MC system, they provide a significant improvement in terms of ISI mitigation. Furthermore, inspired by the biological behaviour of MC system, different types of molecules with resistance or kill effect on information carrying molecules have also been proposed as another method to overcome the ISI effect [1]. Yet, this technique requires extra resources and intelligence to handle the resistance molecular type and tends to increase the amount of molecules in the medium most likely creating collision.

Error correction codes (ECC) have been proposed as an alternative way to mitigate the adverse effects of ISI in MC systems by providing error correction capability at the receiver. Hence, ECC can results in easy manipulation of the system compared to using resistance molecules to overcome ISI. More specifically, the bit error performance of established codes, such as Hamming codes (HC) [5], Euclidean Geometry Low Density Parity Check, and Cyclic Reed-Muller codes [5], have been applied and investigated in MC systems. A new type of ECC, called ISI free code, was proposed and designed to prevent occurrence of ISI via the predefined redundant bit patterns in MC systems [6]. Furthermore, in [7], the authors concentrate on improving the bit error performance via selforthogonal convolutional codes, which takes into account the energy requirements at the receiver for decoding the error correction information.

Different from existing literature, we introduce the ReedSolomon (RS) codes for error correction in the point-to-point $\mathrm{MC}$ systems with a point transmitter and a full absorption 


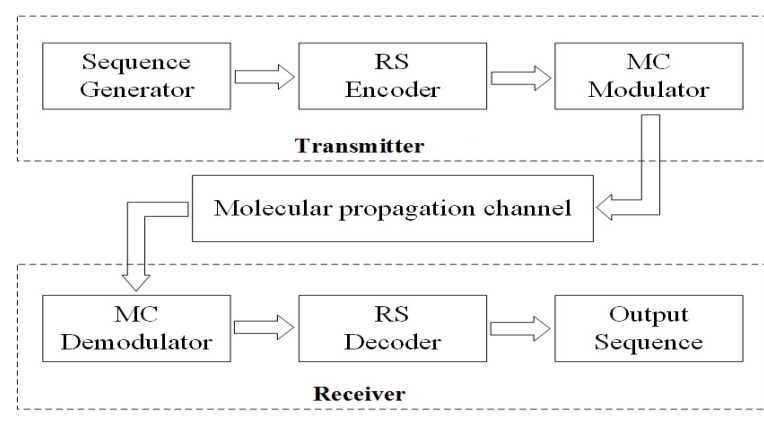

Fig. 1. Block Diagram of the Proposed MC system

receiver. The RS codes are non-binary block codes and are highly effective against burst and random errors in many real channels [8] compared to $\mathrm{HC}$ that only capable of correcting single bit error. Also RS code is one of the few forward error correction code, which can attain the theoretical limit known as the Singleton bound. Since, RS codes utilize the symbol based arithmetic, it can decode codewords with longer block lengths with less decoding time, compared to HC [8]. Another advantage of RS codes is the existence of efficient decoding algorithm due to vast applications of RS codes in data communication. More importantly, simplified RS codes are relatively easy to implement in terms of hardware [9].

In this paper, we present an analytical model for the point-to-point MC systems with RS codes and derive an expression for the approximated bit error probability (BEP) of the proposed system. Furthermore, the derived analytical result is verified by the proposed simulation framework, which captures the random Brownian motion of each molecule in the diffusion-based MC system. The results obtained from simulation are in close agreement with the analytical values. Furthermore, it is observed that the BEP performance is greatly improved via RS codes, and the performance gain increases with the increase of the number of molecules per bit and the minimum distance of the codeword. The remainder of the paper is organized as follows. Section II presents the system model, and Section III briefly introduce RS codes. Section IV presents an expression for the BEP of the proposed MC system. Section V evaluates the performance improvement achieved by the proposed RS codes using numerical and simulation results. Conclusions are given in SectionVI.

\section{SYSTEM MODEL}

We consider a point-to-point diffusion-based MC system with a full absorption receiver with radius $r$ and a point transmitter located distance $d$ away from the surface of the receiver. The block diagram of the proposed MC system is outlined in Fig. 1. In the diffusion-based MC system, the information is modulated on the number of the molecules emitted by the transmitter at start of each bit interval, $T_{b}$. Given that the molecules are released into a medium of large extent compared to their size, collisions between messenger molecules are neglected. Each molecule randomly diffuses in the medium following Brownian motion, with constant diffusion coefficient $D$. Once a molecule reaches the surface of the receiver, it will be absorbed by the receptors at the surface of the receiver. The process of messenger molecules hitting the body of the receiver is named as the hitting process.

For the modulation scheme, we adopt the Binary Concentration Shift Keying (BCSK) as in [2]. At the transmitter, the information is conveyed through the number of molecules being emitted at the start of each bit interval. At the receiver, if the total number of molecules absorbed during a bit interval is above a threshold, the received bit is demodulated as 1 , and otherwise as 0 . To facilitate error correction, RS encoded redundant data is embedded into the message bit pattern at the transmitter. These extra data is utilized at the receiver to recover from bit errors, in turn improving the overall BEP.

\section{ReEd Solomon Codes}

In communication theory, ECC is used for error detection and correction to achieve an acceptable level of accuracy for the received information, when data is transmitted through error prone transmission channels. As one type of ECC, the Reed Solomon codes was first proposed by I. S. Reed and G. Solomon, which is a non-binary Bose, Chaudhuri, and Hocquenghem $(\mathrm{BCH})$ code, with a simple algorithm for the error detection and correction [9]. Considering that the RS codes are well suited for correcting burst errors, they are widely used in digital data transmission and storage applications. We denote RS code with $n$ length codeword and $k$ length input message block, as $R S(n, k)$ and it is defined over a Galois Field $\left(G F\left(p^{m}\right)\right)$, where $p$ is a prime, and $m$ is a positive integer. The length of the parity bits is $n-k$ bits, which has the capacity to correct up to $\frac{n-k}{2}$ number of errors per codeword [9].

The RS codes can be implemented very easily at the encoder using Linear Feedback Shift Registers and at the decoder using Berlekamp algorithm. The systematic RS encoder, appends parity information to the original message in such a way that the constructed codeword is completely divisible by the generator polynomial using Galois Field algebra. This generator polynomial is shared by both the RS encoder and decoder.

The RS decoding operates in two main stages. In the first stage, the decoder inspects the received codeword for errors. The received codeword is identified as in error, if it is not completely divisible by the generator polynomial. This technique is called syndrome computation. If syndrome calculation results in zero, the decoder terminates, otherwise in the second stage, the system attempts to correct the error by detecting the error position and error value. To correct the identified errors, the decoder first determines the error locator polynomial using either Berlekamp-Massey algorithm [10] or Euclidean algorithm [10]. The former leads to a more efficient implementation, while the latter is easier to implement. In the next stage, the Chien search algorithm [10] is used to solve the roots of the error locator polynomial gernerated, which indicate the error locations in the receieved codeword. Then, Forney algorithm [10] is used to estimate the error magnitudes. Once the error location and the error values are estimated, then a correction is applied to the received codeword to recover from the error. In depth description of each algorithm used in the implementation of RS codes can be found in [10] while RS 
decoder implementation presented in this paper can be found in [9].

One of the key bottlenecks in the implementation of ECC in $\mathrm{MC}$ environment is the complexity and the processing power requirements. Inspired by the extensive application of RS codes in battery powered devices, many research are conducted on optimizing the energy performance of the RS coders. For instance, it is reported in [11] that the energy consumption of RS coding can be reduced by $40 \%$ in low activity environments and can be synthesized by using $45 \mathrm{~nm}$ technology. The reduced complexity algorithms with acceptable level of bit error rate are another method adopted by many researchers to achieve low complexity and low power requirements. For instance, [12] proposes to apply the soft information based decoding to achieve better results in error correction rates compared to hard decision decoding, while keeping the complexity at lower level. In summary, it is safer to assume that RS codes can be implemented with reduced complexity in MC with the advanced research on low complexity implementation of RS coders.

\section{BIT ERROR PROBABILITY}

It is known that in $\mathrm{MC}$, the number of absorbed molecules during one bit interval, $T_{b}$, can be modeled as binomial distribution. For computational tractability, the binomial distribution can be approximated using the normal distribution, when $N_{m}$ the number of molecules sent at the start of each bit interval is large, and $P_{h i t}(d, t)$ the fraction of molecules absorbed by the receiver is not near 0 or 1 . Thus, we express the total number of absorbed molecules, in the $i^{\text {th }}$ bit $N_{h i t}[i]$, during $\left[(i-1) T_{b}, i T_{b}\right]$ in the absence of ISI as

$N_{\text {hit }}[i] \sim \mathscr{N}\left(N_{m} P_{h i t}\left(d, T_{b}\right), N_{m} P_{h i t}\left(d, T_{b}\right)\left[1-P_{h i t}\left(d, T_{b}\right)\right]\right)$.

Yet, due to ISI, the actual number of molecules absorbed during $T_{b}$, account for the number of molecules recieved from the current bit $\left(a_{i}\right), N_{a_{i}}$, and from the previous bits, $N_{a_{1: i-1}}$. However, prior work [13] shows that the most prominent effect on ISI is contributed by the nearest past bit, $a_{i-1}$, with an appropriate bit interval, $T_{b}$. As such, in the presence of ISI, the $N_{\text {hit }}[i]$ can be approximated as

$$
N_{h i t}[i]=a_{i} N_{a_{i}}+a_{i-1} N_{a_{i-1}}
$$

where

$$
N_{a_{i}} \sim \mathscr{N}\left(N_{m} P_{h 1}, N_{m} P_{h 1}\left[1-P_{h 1}\right]\right)
$$

and

$$
\begin{aligned}
N_{a_{i-1}} \sim & {\left[\mathscr{N}\left(N_{m} P_{h 2}, N_{m} P_{h 2}\left[1-P_{h 2}\right]\right)\right.} \\
& \left.-\mathscr{N}\left(N_{m} P_{h 1}, N_{m} P_{h 1}\left[1-P_{h 1}\right]\right)\right] .
\end{aligned}
$$

In (3) and (4), $P_{h j}$ denotes $P_{h i t}\left(d, j T_{b}\right)$ and it can be derived as in [1], using Eq. (6), Eq. (7) and Eq. (8).

For an uncoded binary channel, the bit error probability, $P_{e}$, over all possible combinations of $a_{i}$ can be expressed as

$$
P_{e}=P_{r}\left[e \mid a_{i}=0\right] P_{r}\left[a_{i}=0\right]+P_{r}\left[e \mid a_{i}=1\right] P_{r}\left[a_{i}=1\right],
$$

This can be further expanded by considering the possible outcomes for both $a_{i}$ and $a_{i-1}$ as

$$
\begin{aligned}
P_{e} & =P_{r}\left[e \mid a_{i}=0, a_{i-1}=0\right] \cdot P_{r}\left[a_{i}=0\right] \cdot P_{r}\left[a_{i-1}=0\right] \\
& +P_{r}\left[e \mid a_{i}=0, a_{i-1}=1\right] \cdot P_{r}\left[a_{i}=0\right] \cdot P_{r}\left[a_{i-1}=1\right] \\
& +P_{r}\left[e \mid a_{i}=1, a_{i-1}=0\right] \cdot P_{r}\left[a_{i}=1\right] \cdot P_{r}\left[a_{i-1}=0\right] \\
& +P_{r}\left[e \mid a_{i}=1, a_{i-1}=1\right] \cdot P_{r}\left[a_{i}=1\right] \cdot P_{r}\left[a_{i-1}=1\right]
\end{aligned}
$$

where $P_{r}\left[e \mid a_{i}, a_{i-1}\right]$ is the conditional probability of error for $i^{t h}$ bit with known transmitted bits $a_{i}$ and $a_{i-1}$. The conditional probabilities in equation (6) can be approximated as

$$
\begin{gathered}
P_{r}\left[e \mid a_{i}=0, a_{i-1}=1\right]=P_{r}\left[N_{h i t} \geq \tau\right]=P_{r}\left[N_{a_{i-1}} \geq \tau\right] \\
\approx Q\left(\frac{\tau-\left[\left(N_{m} P_{h 2}\right)-\left(N_{m} P_{h 1}\right)\right]}{\sqrt{\left(N_{m} P_{h 1}\left[1-P_{h 1}\right]\right)+\left(N_{m} P_{h 2}\left[1-P_{h 2}\right]\right)}}\right) \\
P_{r}\left[e \mid a_{i}=1, a_{i-1}=0\right]=P_{r}\left[N_{h i t}<\tau\right]=P_{r}\left[N_{a_{i}}<\tau\right] \\
\quad \approx 1-Q\left(\frac{\tau-\left(N_{m} P_{h 1}\right)}{\sqrt{N_{m} P_{h 1}\left[1-P_{h 1}\right]}}\right) \\
P_{r}\left[e \mid a_{i}=1, a_{i-1}=1\right]=P_{r}\left[N_{h i t}<\tau\right]=P_{r}\left[N_{a_{i}}+N_{a_{i-1}}<\tau\right] \\
\approx 1-Q\left(\frac{\tau-\left(N_{m} P_{h 2}\right)}{\sqrt{\left(2 N_{m} P_{h 1}\left[1-P_{h 1}\right]\right)+\left(N_{m} P_{h 2}\left[1-P_{h 2}\right]\right)}}\right)
\end{gathered}
$$

and

$$
P_{r}\left[e \mid a_{i}=0, a_{i-1}=0\right]=P_{r}\left[N_{\text {hit }} \geq \tau\right]=0
$$

where $\tau$ is the detection threshold and $Q($.) denotes the tail probability of the standard normal distribution.

The evaluation of the post-decoding BEP of block codes, $P_{b}$, is complex, even classical coding books like [9] and [10] fail to present a closed-form expression. As such, bounds for post-decoding BEP of block codes can be obtained as

$$
\frac{1}{k} P_{w} \leq P_{b} \leq P_{w}
$$

following [10], where $P_{w}$ represents the block error probability. When the complete weight distribution of the specific $R S(n, k)$ code is not available, the upper bound is used as the post-decoding BEP. As such, we approximate the postdecoding BEP of RS codes as [10]

$$
P_{b}=\sum_{i=t_{e}+1}^{n}\left(\begin{array}{l}
n \\
i
\end{array}\right) P_{e}^{i}\left(1-P_{e}\right)^{n-i}
$$

where $t_{e}=\frac{n-k}{2}$ and $P_{e}$ is the bit error probability for the uncoded channel. Substituting (7), (8), (9), and (10) into (6), we obtain the $P_{e}$ in (12).

\section{Performance Evaluation}

\section{A. Simulation Framework}

To validate the expression of BEP derived in Section IV, we present particle-based simulation framework extended from [2] and [14]. This simulation framework takes into account the bit pattern generation, the RS encoding, the signal modulation, the propagation, the molecule reception, the signal demodulation, and the RS decoding as outlined in Fig. 1. We simulate the $2 \mathrm{D}$ environment due to the computational complexity in 


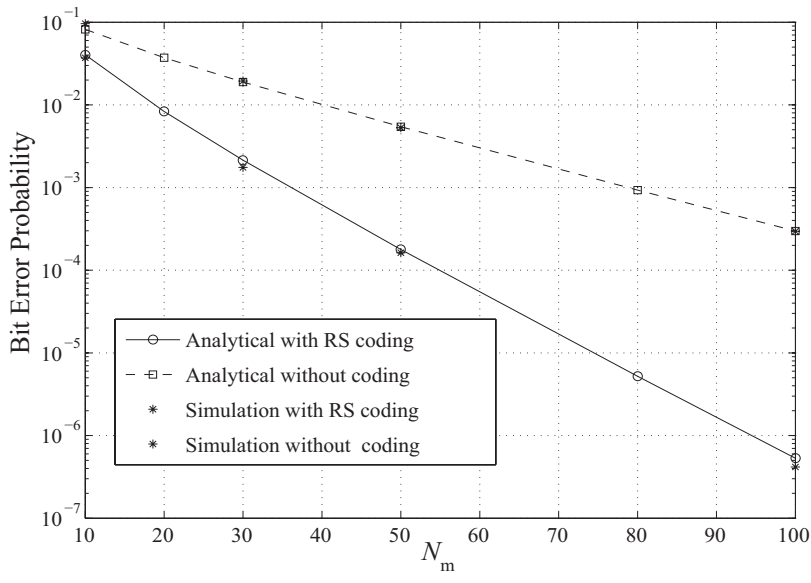

Fig. 2. Bit error probability of RS $(4,2)$ coded and uncoded bit stream.

particle-based simulation for 3D MC system with RS codes. The information bits are randomly generated with simulation parameters set as: $d=1 \mu \mathrm{m}, D=79.4 \mu^{2} / \mathrm{s}, T_{b}=0.032 \mathrm{~s}$, $\tau=\frac{N_{m}}{2}$, and the length of the bit sequence is 2400000 with 100 repetitions per each iteration. The analytical results are plotted using (12), with the assumption that the occurrence of bit 1 and 0 of the transmitted bit is equally likely.

\section{B. Numerical Results}

In this subsection, we present the simulation and analytical results of the bit error probability of our proposed MC system. The accuracy of the theoretical BEP approximation is evaluated. Fig. 2 plots the impact of RS coding on the BEP of proposed MC system. It is shown that the theoretical results match with that of particle-based simulations, which validate the accuracy of our derived expression. Compared with uncoded case, the BEP is greatly improved with the help of $\mathrm{RS}(4,2)$ applied in our proposed MC system. For instance, with $N_{m}=100$, the BEP achieved by the RS $(4,2)$ is $5 \times 10^{-6}$, which is a negligible level.

Fig. 3 plots the BEP of the proposed system with various minimum distance of the RS code and HC. According to Fig. 3 , RS codes is shown to provide a significant level of BEP improvement compared to HC. We also observe that the BEP of the RS codes can be greatly improved by increasing the minimum distance and the coding gain, $\frac{k}{n}$. This is because increasing $n-k$ increases the error correction capacity of the RS code given by $\frac{n-k}{2}$, in comparison to $\mathrm{HC}$ which is only capable of correcting single bit error. In addition, since particle-based simulations for very large input bit streams are time consuming, only analytical results are presented.

\section{CONCLUSION}

In this paper, we introduced RS code for error correction of the diffusion-based MC system. We derived the bit error probability of the proposed MC system with full absorption receiver using theoretical approximations. The derived analytical results for the bit error probability are verified by particlebased simulations. It is shown that the RS code substantially

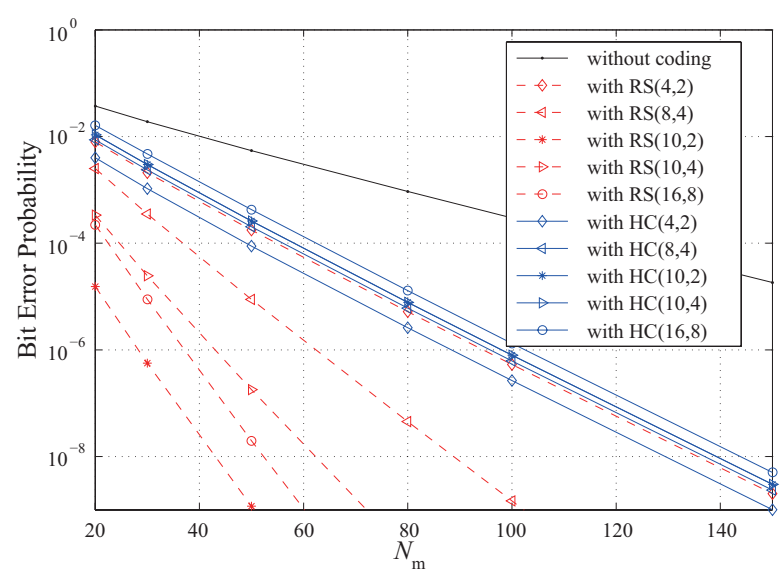

Fig. 3. Bit error probability of RS codes and Hamming Codes (HC).

improves the bit error probability of MC systems, compared to uncoded MC systems. The bit error probability can be further enhanced by increasing, the number of molecules emitted per bit and the minimum distance of the RS codeword. These results justify the effectiveness of RS codes in diffusionbased MC systems. The extension of this work under adaptive weighted threshold detection scheme and finding the optimum combinations of $(n, k)$ can be considered in future work.

\section{REFERENCES}

[1] N. Farsad, H. B. Yilmaz, A. W. Eckford, C. Chae, and W. Guo, "A Comprehensive Survey of Recent Advancements in Molecular Communication," IEEE Commun. Surv. Tutorials, pp. 1-29, Feb. 2016.

[2] Y. Deng, A. Noel, M. Elkashlan, A. Nallanathan, S. Member, and K. C. Cheung, "Modeling and Simulation of Molecular Communication Systems with a Reversible Adsorption Receiver," IEEE Trans. Mol. Biol. Multi-Scale Commun., vol. 1, no. 4, pp. 347 - 362, Dec. 2015.

[3] Y. Deng, W. Guo, A. Noel, A. Nallanathan, and M. Elkashlan, "Enabling energy efficient molecular communication via molecule energy transfer," IEEE Communications Letters, vol. 21, no. 2, pp. 254-257, Feb. 2017.

[4] Y. Deng, A. Noel, W. Guo, A. Nallanathan, and M. Elkashlan, "3d stochastic geometry model for large-scale molecular communication systems," in IEEE Glob. Telecommun. Conf., Dec. 2016, pp. 1-6.

[5] Y. Lu, M. D. Higgins, M. S. Leeson, and S. Member, "Comparison of Channel Coding Schemes for Molecular Communications Systems," IEEE Trans. Commun., vol. 63, no. 11, pp. 3991-4001, Nov. 2015.

[6] P. J. Shih, C. H. Lee, and P. C. Yeh, "Channel codes for mitigating intersymbol interference in diffusion-based molecular communications," in IEEE Glob. Telecommun. Conf., Dec. 2012, pp. 4228-4232.

[7] Y. Lu, M. D. Higgins, and M. S. Leeson, "Self-orthogonal convolutional codes (SOCCs) for diffusion-based molecular communication systems," in IEEE Int. Conf. Commun., no. 1, Jun. 2015, pp. 1049-1053.

[8] G. Mitchell, "Investigation of Hamming , Reed-Solomon , and Turbo Forward Error Correcting Codes," Army Research Laboratory, Adelphi, ARL-TR-4901, Tech. Rep. Jul., 2009.

[9] S. Wicker and V. K. Bhargava, Reed-Solomon Codes and Their Applications. IEEE Press, 1994.

[10] S. Lin and D. J. J. Costello, Error Control Coding: Fundamentals and Applications., 2nd ed. Pearson Prentice-Hall, Jun. 2004.

[11] J. D. Allen, "Energy Efficient Adaptive Reed-Solomon Decoding System," Ph.D. dissertation, University of Massachusetts, Jan. 2008.

[12] N. Wehn, S. Scholl, P. Schläfer, T. Lehnigk-Emden, and M. Alles, Challenges and Limitations for Very High Throughput Decoder Architectures for Soft-Decoding. Springer International Publishing, 2015, pp. 7-31.

[13] M. S. Kuran, H. B. Yilmaz, T. Tugcu, and B. Özerman, "Energy model for communication via diffusion in nanonetworks," Nano Commun. Netw., vol. 1, no. 2, pp. 86-95, Jul. 2010.

[14] W. Guo, Y. Deng, B. Li, C. Zhao, and A. Nallanathan, "Eavesdropper localization in random walk channels," IEEE Communications Letters, vol. 20, no. 9, pp. 1776-1779, Sep. 2016. 